\title{
ANALYSING THE CHINESE STOCK MARKET USING THE HURST EXPONENT, FRACTIONAL BROWNIAN MOTION AND VARIANTS OF A STOCHASTIC LOGISTIC DIFFERENTIAL EQUATION
}

\author{
O. VUKOVIC \\ University of Liechtenstein, Liechtenstein.
}

\begin{abstract}
The Chinese stock market is rapidly developing and is becoming one of the wealth management investment centres. Recent legislation has allowed wealth management products to be invested in the Chinese stock market. By taking data from St. Louis Fed and analysing the Chinese stock market using the Hurst exponent, which was calculated by using two methods, and fractional Brownian motion, it is proved that the Chinese stock market is not efficient. However, further analysis was directed to finding its equilibrium state by using logistic difference and a differential equation. To achieve more precise movement, a stochastic logistic and delayed logistic differential equation have been implemented which are driven by fractional Brownian motion. As there is no explicit solution to a delayed differential equation using the Stratonovich integral, a method of steps has been used. A solution has been obtained that gives the equilibrium state of the Chinese stock market. The following solution is only for a Hurst exponent that is higher than $1 / 2$. If Hurst exponent is close to $1 / 2$, then Brownian motion is an ordinary one and the equilibrium solution is different. Sensitivity analysis in that case has been conducted in order to analyse possible stationary states. The main conclusion is that the Chinese stock market is still not mature enough to be efficient, which represents a hidden risk in wealth management investing. A new approach to valuing the efficiency of the market by using nonlinear dynamics and system analysis has been proposed. Keywords: Chinese stock market, fractional Brownian motion, Hurst exponent, stochastic logistic differential equation.
\end{abstract}

\section{INTRODUCTION}

Wealth management products (WMP) are bank-generated investment products that are sold to their retail and institutional customers. Similar to money market funds, WMPs pool funds with a relatively short investment horizon (as short as 5 days) and invested them in longer duration assets to arbitrage the difference in returns. Disclosure on these products, including what assets they invested in and the likely returns of each, is limited. WMPs essentially circumvent China's tight control of interest rates by rewarding investors' higher returns than deposit rates. Recently, it has been allowed Chinese banks to invest money directly to stock and bond markets. It is the aforementioned decision that could have high potential consequences for the Chinese middle market as the stock and bond market is characterised by nonlinearities and, therefore, nonlinear dynamics can be applied to prove that Chinese stock market is characterised by nonlinear model that is more effective than the Bachelier's random walk theory based on stochastic models. The complex stochastic behaviour of a chaotic system is determined by the nonlinear equations of a determined system that has finite independent free variables, and the phase space is a finite dimension status space that is constructed by these independent variables. To analyse the system, the level of noise must be analysed. It is tried to subject stock price movement of Chinese market to nonlinear analysis. Hurst exponent is presented and its analysis is conducted to analyse nonlinearity level in the Chinese stock market. At the same time, there is a connection between Hurst exponent and fractional Brownian motion. If the movement of Chinese stock market can be explained by using fractional Brownian motion or Gaussian process, it can be concluded that market is not predictable and inefficient. Fractional Brownian 
motion is characterised by a non-semimartingale process and therefore cannot be integrated. However, if Hurst exponent has a value of $1 / 2$, then Brownian motion is characterised as semimartingale and as it is possible to integrate with some type of stochastic integral, it is then possible to prove that the movement of Chinese stock market can be decomposed to a local martingale and cadlag-adapted process of bounded variation. As the definition adapted process is that it cannot be predicted, it is obvious that the value of Hurst exponent of $1 / 2$ causes Chinese stock market to be unpredictable. Before going to real analysis and generated results, background concerning stochastic analysis as well as Hurst exponent will be presented. Main intention is to present the fact that investing in Chinese stock and bond markets can present risks for wealth management in China because if market is inefficient, it can be proved that according to ergodic theory, it is impossible to predict its state. Although according to Hairer [1], there are two theorems that state, if Hurst parameter has approximate value of $1 / 2$, it can be predicted that the solution of stochastic differential equation converges to stationary value taking into consideration some initial conditions. As stochastic differential equations entail Brownian motion, therefore as fractal Brownian motion is being considered, it is possible that by using some form of stochastic differential equation, it can be proved that the movement of Chinese stock market converges to some specific value. To prove the following assumption, delayed logistic equations is used. It is known that ordinary logistic equation is being used to model population dynamics. In this paper, first logistic equation is being analysed. After that, Brownian motion is implemented in delayed logistic equation and approximate solution is presented. As stochastic analysis presents Ito integral and Stratonovich integral, it is presented that equation using Stratonovich integral cannot be solved. As ordinary logistic difference equation is characterised by cobweb diagram and attractor, it is tried to find a solution for delayed logistic equation and find its attractor and equilibrium value. Afterwards, delayed stochastic differential equation including Brownian motion is included. Its approximate solution is found and it is simulated to find the equilibrium point which is compared with the current state of Chinese stock market in order to see if it attains its equilibrium value. The results are discussed afterwards. In this manner, risk is analysed to see if wealth management has one of the global risks and that is investing in a market which movement cannot be predicted. Although the aforementioned approach is really unconventional, some results are really interesting and hopefully the provided results will be helpful to forward research in the following direction. Depending on the value of Hurst exponent, it can be concluded that if there is a high value of standard deviation and Hurst exponent value is $1 / 2$, delayed stochastic logistic equation is used to model movement of stock price. It will be showed that because of high volatility, it is very hard to predict stock price movement on the Chinese stock market.

\section{BACKGROUND}

Fractional Brownian motion is defined as continuous Gaussian process with $B_{H}(t)$ on $[0, T]$, which starts at 0 , has expectation 0 for all $t$ in $[0, T]$, and has the following covariance function [3]:

$$
E\left[B_{H}(t) B_{H}(s)\right]=\frac{1}{2}\left(|t|^{2 H}+|s|^{2 H}-|t-s|^{2 H}\right)
$$

where Hurst exponent has the value between $(0,1)$. The value of Hurst exponent determines what kind of movement will the time series exhibit. Hurst exponent can have the value between $(0,1)$. If Hurst exponent is higher than $1 / 2$, then time series exhibit positive correlation; if Hurst exponent has the value less than $1 / 2$, then the series exhibits negative correlation, while for the value of Hurst exponent of $1 / 2$, series exhibits ordinary Brownian motion. To introduce the stochastic differential equation, few assumptions will be made: 
1. Stability;

2. Non-degeneracy;

3. Growth and regularity.

2.1 Stability

$$
\begin{gathered}
C^{\left(A_{1}\right)}>0, \\
\langle f(x)-f(y), x-y\rangle \leq \min \left\{C_{1}^{\left(A_{1}\right)}-C_{2}^{\left(A_{1}\right)}\|x-y\|^{2}, C_{3}^{\left(A_{1}\right)}\|x-y\|^{2}\right\}
\end{gathered}
$$

for every $x, y \in R_{n}$ stability is the only condition used to limit the movement in a time series.

\subsection{Growth and regularity}

There exist constants $C, N>0$ such that $f$ and its derivatives satisfy the following condition:

$$
\|f(x)\| \leq C\left((1+\|x\|)^{N} \quad\|D f(x)\| \leq C\left((1+\|x\|)^{N}\right.\right.
$$

For every $x \in R^{n}$ this condition only pertains to the limited growth of model that is controlled by the constant and parameter $N$. The derivative says that the growth of the function is limited and is given in one determined range.

\subsection{Non-degeneracy}

The $n \times n$ matrix of $\sigma$ is invertible.

As we will be considering the SDEs, it is recommendable to limit to the following equations of the form:

$$
\mathrm{d} x_{t}=f\left(x_{t}\right) \mathrm{d} t+\sigma \mathrm{d} B_{H}(t)
$$

where $x_{t} \in R^{n}, f: R^{n} \rightarrow R^{n}$, and $B_{H}$ is an $n$-dimensional FBM with Hurst parameter that will be analysed. $H$ and $\sigma$ are constant and invertible $n \times n$. This should be interpreted as an integral equations. For the Hurst parameter of $1 / 2$, the following equation becomes ordinary stochastic differential equation as Hurst parameter of $1 / 2$ for fractional Brownian motion proves that Brownian motion is semimartingale and it behaves as random walk. FBM is self-similar with the scaling law

$$
t \rightarrow B_{H}(a t) \approx t \mapsto a^{H} B_{H}(t)
$$

It appears that FBM can be used to model long-time correlations in stock markets. The reason for choosing fractional Brownian motion for modelling is that the value of Hurst exponent higher than $1 / 2$ presents genuine long-term correlations that persist even under rescaling. Also, there exists simple formula that relates fractional Brownian motion to standard one, and there this is a useful result.

When Hurst exponent has higher value than $1 / 2$, more regularity for $f$ is required and it will be assumed that the stronger condition for growth and regularity holds.

The derivative of $f$ is globally bounded. 
Hairer in his paper on 'Ergodicity of stochastic differential equations driven by fractional Brownian motion' proves that the above-defined stochastic differential equations possess a unique stationary solution. Two theorems will be quoted, for detailed proof look at the Hairer aforementioned paper $[1,5,6]$ :

$T 1$ (Small Hurst parameter). Let $H \in(0,1 / 2)$ and let $f$ and $\sigma$ satisfy the above-presented conditions. Then, for every initial condition, the solution to stochastic differential equations converges toward a unique stationary solution in the total variation norm. The difference between stationary solution is bounded by $C_{\gamma} t^{-\gamma}$ for large $t$ for every $\gamma<\max _{\alpha<\mathrm{H}} \alpha(1-2 \alpha)$.

$T 2$ (Large Hurst parameter). Let $H \in(1 / 2,1)$ and let $f$ and $\sigma$ satisfy the above-presented conditions. Then, for every initial condition, the solution to stochastic differential equations converges toward a unique stationary solution in the total variation norm. The difference between stationary solution is bounded by $C_{\gamma} t^{-\gamma}$ for large $t$ for every $\gamma<\frac{1}{8}$.

The uniqueness of the stationary solution pertains to the class of stationary solution adapted to the natural filtration induced by the two-sided fractional Brownian motion that drives the equation. To analyse stock market, it is going to be assumed that the movement is based only on past information, not the future ones. If the movement would be based on future solutions, there would be in theory other stationary solutions. Analysing the case of $H=\frac{1}{2}$, it is well known that the convergence to the stationary state is exponential. The total variation refers between measures on the space of paths. A stock market can be considered as a system driven by Markovian noise. Only complete, metrizable and separate space will be considered. Because processes are non-Markovian, it is necessary to know its past state, not only the present one. Markov process is the defined in the following manner.

Let $(\Omega, \mathcal{F}, \mathcal{P})$ be a probability space with a filtration $\left(\mathcal{F}_{s}, s \in I\right)$, for some (totally ordered) index set $I$; and let $(S, S)$ be a measure space. An $S$-valued stochastic process adapted to the filtration is said to possess the Markov property with respect to the $\left\{\mathcal{F}_{t}\right\}$ if, for each $A \in S$ and each $s, t \in I$ with $s<t$,

$$
P\left(X_{t} \in A \mid F_{s}\right)=P\left(X_{t} \in A \mid X_{s}\right)
$$

A Markov process is a stochastic process, which satisfies the Markov property with respect to its natural filtration. Non-Markovian process is defined in the same manner, but by including past state. It means that stochastic process has got memory property, because it remembers the past states.

To proceed, Hurst exponent must be defined. It is used to analyse long memory in time series. It relates to the autocorrelation time series and it is defined in terms of the asymptotic behaviour. It has the following formula:

$$
\mathrm{E}\left[\frac{R(n)}{S(n)}\right]=C n^{H} \text { as } n \rightarrow \infty
$$

where $R(n)$ is the range of the first $n$ values, $S(n)$ is their standard deviation, $\mathrm{E}[x]$ is the expected value, $n$ is the time span of the observation (number of data points in a time series), and $C$ is a constant.

Hurst exponent can be estimated in various ways and it is really hard to be estimated. Some numerical methods can be used that can give approximate values to the aforementioned Hurst exponent.

To proceed with analysis, stochastic logistic differential equation with implemented fractional Brownian motion will be presented. It will be analysed and the solution will be presented.

First ordinary logistic map will be presented. It has the following form:

$$
x_{n+1}=r x_{n}\left(1-x_{n}\right)
$$


where $x_{n}$ is a number between zero and one that represents the ratio of existing population to the maximum possible population. It is obvious that for the equation where $r$ is $1.2, x_{0}$ has the value of 0.4 . It is obvious that attractor point exists. It should also be noted that when $r$ changes, the attractor point changes. In our case, we are speaking about dynamic $r$ which changes continuously, so the equilibrium value changes. Delayed logistic equation looks like the following:

$$
x^{\prime}(t)=\operatorname{ax}(t)(1-x(t-\tau))
$$

where $\tau$ is delay parameter. For a $\tau<1 / e$, solutions are monotonic. For $1 / e<\alpha \tau<\pi / 2$, the solutions are oscillatory and asymptotically approach $x=1$. For $\alpha>\pi / 2$, the solutions approach a limit cycle. But when considering stock market movement in China, parameter $\alpha$ changes constantly as well as delayed parameter. By considering the dynamic movement for values of $\alpha=1.74566$ and delay parameter of $\tau=1.42783$, we get high oscillation that are continuous. If it is possible to predict the movement of $a$ in the other words, its minimum and maximum then it is possible to predict movements, but to be more precise, we make the logistic equation stochastic one. The following representation is obtained [4]:

$$
d x(t)=x(t) \times\left(1+x(t) / x_{\max }\right) \times(r d t+\sigma d w(t))
$$

where $r$ is the growth rate dependent of $t, \sigma$ is the volatility (in example of the stock market) and $w(t)$ is the standard Brownian motion with Hurst exponent of 1/2. Volatility changes can be given a range, for which delayed stochastic logistic equations will be used.

It is obvious that logistic equation which was simulated achieves its equilibrium value of 4 , because parameters used are $r=1, x_{\max }=4, x_{0}=1, \sigma=1 / 2$. It is seen that even for oscillations of $1 / 2$, there are many possibilities of oscillation of the value using ordinary Brownian motion.

\section{METHODOLOGY AND MODELLING}

First, data for calculations were taken from the St. Louis FED and total shares prices for all shares of China were taken. By using Hurst exponent and two different packages in R, the values shown in Fig. 1 are obtained.

The following equation proves that the average Hurst 48-month value is around 0.48 , so we can assume that it is close to 0.5 which mimics the ordinary Brownian motion. However, other package

\section{Hurst Index Comparison for SPASTT01CNM661N}

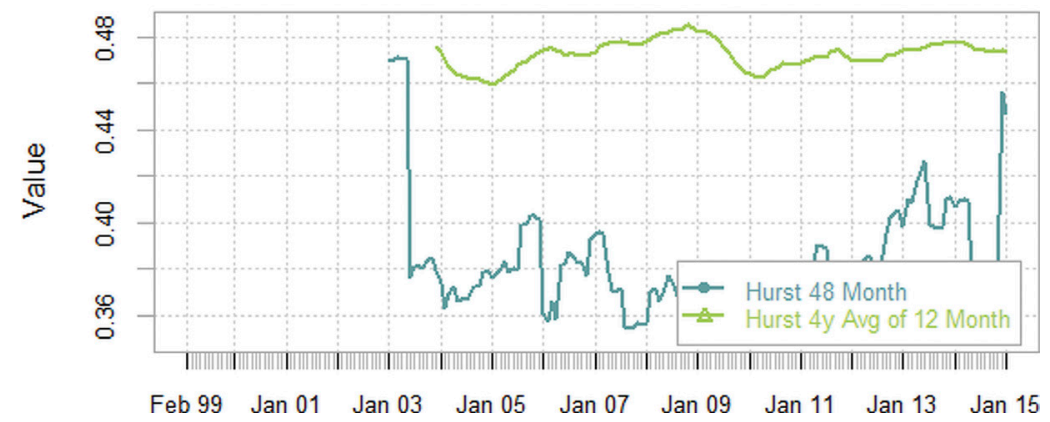

Figure 1: Hurst exponent calculated using $\mathrm{R}$ package. 
(FRN package R) gives the value of 0.66 . Therefore, both values will be analysed to be more precise and find the equilibrium value. If Hurst exponent has the value of 0.5 , then it is assumed that Chinese stock market follows ordinary Brownian motion. Taking into consideration volatility, that is taken from St. Louis FED, the following values are obtained (Fig. 2). Volatility stock price index represents the standard deviation of China stock prices in 365 days. It is obvious that stock price index is really high. Average stock price volatility is $27.5 \%$. However, the calculated value is lower than expected; therefore, we take the latest volatility level which is $30 \%$. The abnormal standard deviations are weighted and they have been weighted to mitigate their effect. Having average stock price volatility, average growth rate should be calculated. To get the current state of index, the latest growth rate should be taken, because only the latest data should be taken into account. The growth rate taken was $29 \%$. The stock price volatility is taken to be $30 \%$. All the aforementioned data are taken pertaining to Shanghai stock index and its recent movements. It is higher than average and it should be taken approximately, because it is also the dynamic variable. Dynamic variables are continuously changing; therefore, only the latest values should be taken and should be changed on a constant basis to get the newest predictions.

The following values have been implemented in the stochastic logistic differential equation presented above and Fig. 3 has been obtained:

Figure 3 demonstrates the movement of Shanghai stock index for the period of April 2014 until February 2015. The average growth rate for this period was calculated. Its value is $12 \%$. It was implemented in the above-presented stochastic differential equation and the result was obtained. Maximum value is considered to be 3500 index points, its beginning value for this period is 2100 . If assumptions are correct, index will reach the aforementioned boundary and then will return to its equilibrium value which is around 3000 (according to the picture). This is expected to happen in 20 months time and interval of equilibrium value is 3000-3100. Attention must be directed that this is a dynamic model, growth is changing constantly as well as standard deviation while beginning value and maximal value must be presumed. Although logistic growth model will always eventually attain the aforementioned value, it is important to emphasise the different paths that fluctuating stock index could follow before achieving that value. At the same time, it should be noted that this dynamical model could only work uptrending markets and it can be used to predict possible resistance levels.

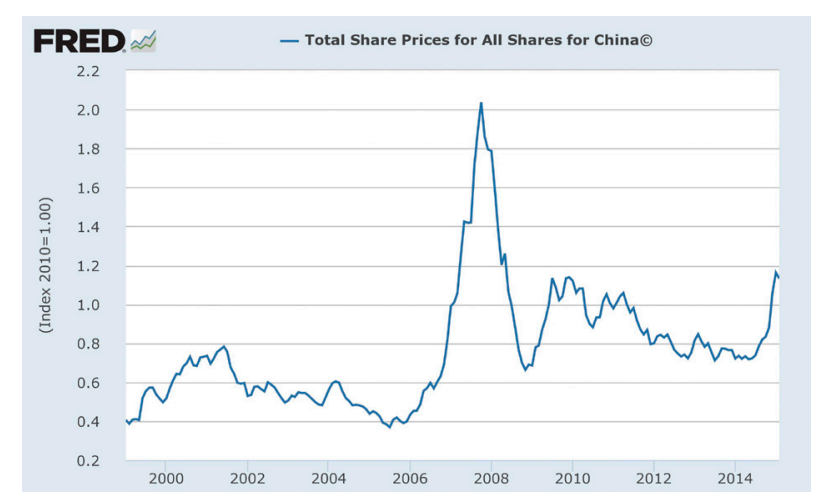

Figure 2: World Bank, volatility of Stock Price Index for China [DDSM01CNA066NWDB], retrieved from FRED, Federal Reserve Bank of St. Louis. 


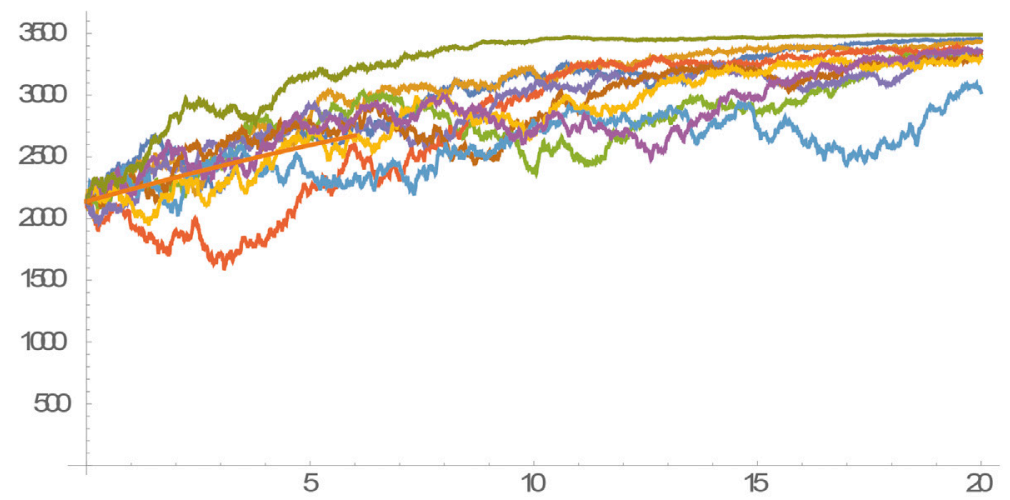

Figure 3: Stochastic logistic equation with parameters for the period April 2014 to February 2015.

Different approach when Hurst index $>1 / 2$. Now the situation will be analysed according to the Hurst index value of 0.66 . The stochastic logistic delayed equation cannot be solved for the Hurst value of 0.66 , because then fractional Brownian motion exists. However, according to Nguyen Tien Dung, the following theorem is proved [2] and his paper On Delayed Logistic Equation using Fractional Brownian motion.

T3. Suppose that $\mu(t)$ is an adapted stochastic process and fulfils the following conditions:

$$
\begin{gathered}
\sup _{0<t<T}\left(e^{\int_{0}^{t} \mu(s) \mathrm{d} s}\right) \in L^{p}(\Omega) \text { for some } p>\frac{1}{H} \\
e^{\int_{0}^{t} \mu(s) \mathrm{d} s} \in L^{q}(\Omega) \text { for some } q>4 \\
\int_{0}^{T}\|\mu(t)\|_{1,4} \mathrm{~d} t<\infty
\end{gathered}
$$

Then, the equation [15]:

$$
\mathrm{d} x_{t}=\mu(t) x_{t} \mathrm{~d} t+\sigma x_{t} \mathrm{~d} W_{t}^{H} \quad X_{0}=x>0
$$

has a unique solution in Polish space, which is given by

$$
X_{t}=X_{0} e^{\int_{0}^{t} \mu(s) \mathrm{d} s+\sigma W_{t}^{H}}
$$

To derive an equilibrium solution, one of the results from Ngyen Tien Dung paper will be used:

For constant $M>0$ such that $\mu(t) \leq M$ for every $t>0$, so we have the following result [7]:

$$
X_{t}=X_{0} e^{\int^{t} \mu(s) \mathrm{d} s+\sigma W_{t}^{H}} \leq X_{0} e^{M t+\sigma W_{t}^{H}}
$$

Taking volatility to be 0.1 and Hurst exponent of $0.66, W_{t} W_{t}^{H}$ is simulated and the results shown in Fig. 4 are obtained [16]: 


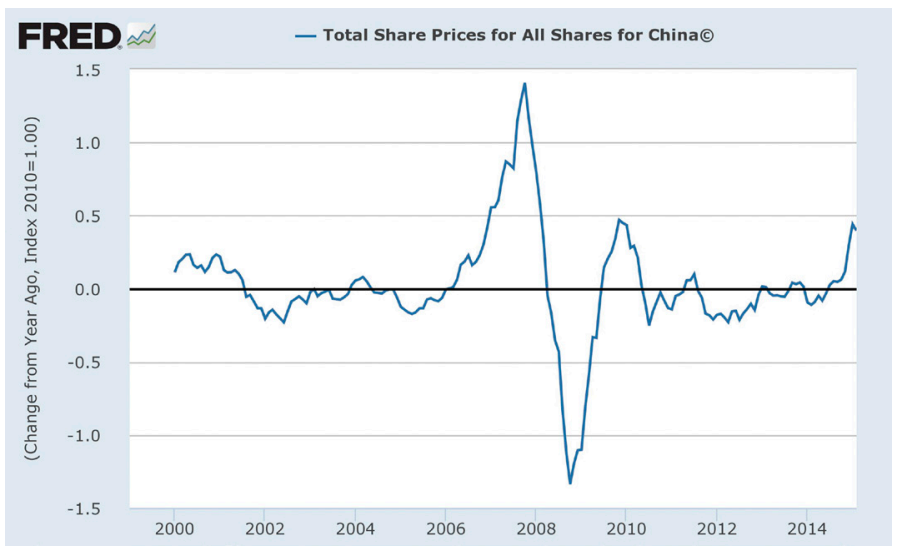

Figure 4: Comparison between total share price China and fBM.

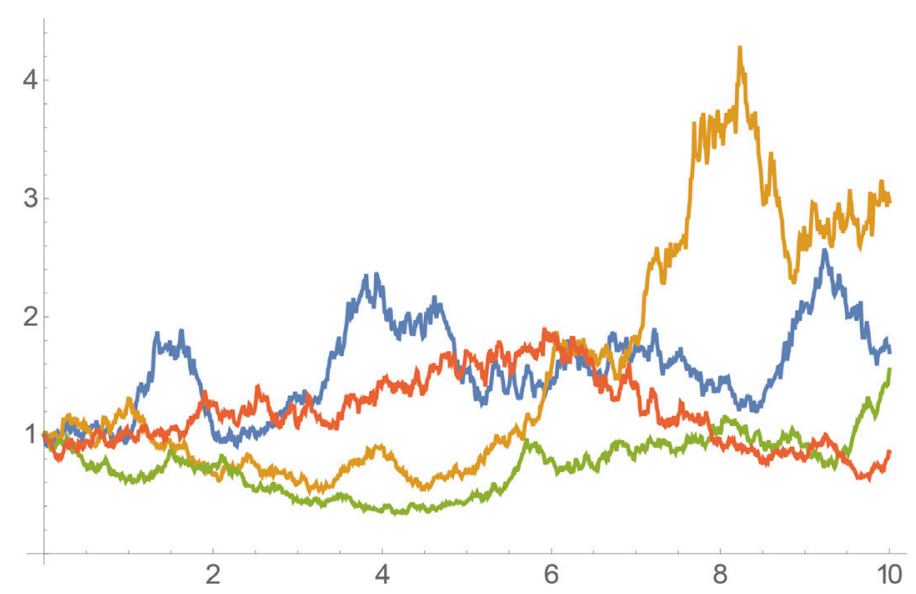

Figure 5: Exponential stochastic equation simulation.

Figure 4 demonstrates that the movement of Chinese stock market can be approximated by fractional Brownian motion $[12,14]$. The next thing that must be conducted is to analyse the possible oscillations of fBM to have approximate range. After running 1000 simulations of fractional Brownian motion, the range of oscillations is from -2 to 2 . Multiplying that by volatility of 0.3 and simulating exponential stochastic motion, the graph shown in Fig. 5 is obtained:

However, the graph can be varied, but approximate solutions are there. Taking the equilibrium value and assuming that average value according to the graph is 1 , we get the following solution $[8,10]$ :

$$
X_{t}=X_{0} e^{\int^{0} \mu(s) \mathrm{d} s+\sigma W_{t}^{H}} \leq X_{0} e
$$

The possible solution taking into consideration the beginning value of index of 2170 and multiplying by $e$, the following result is obtained 5900 which is the highest value index obtained; therefore, it 
marks the index capacity. Again it is confirmed that the beginning value of index is around 2170 even for Hurst exponent of 0.66. The index defined is Shanghai stock index. Results obtained are very interesting as they mimic the movement of Shanghai stock index. The attention should be directed to dynamic hedging as $X_{0}$ is constantly changing [12]. To find and apply the following equations, first the uprising trend must be noticed. The equilibrium value will be the value that is characterised and stationary in the longer period. For example, analysing the Shanghai stock index, it is obvious that its equilibrium value was around 2170 for a longer period and after that it started increasing. Equilibrium value is dynamic and is changing continuous [11], any value that stays for a longer period can be considered equilibrium value and it is implemented in equation as $X_{0}$. This is only valid if calculated Hurst exponent is bigger than 0.5 .

\section{SUMMARY}

Having analysed the Shanghai stock index and Chinese stock market, it has been concluded that its value follows fractional Brownian motion. By implementing stochastic logistic differential equation and calculating Hurst exponent, it has been concluded that it is fairly difficult to predict future movement of stock market in China. To have the right prediction, growth rate that is dynamic and volatility that is also dynamical must be calculated continuously. At the same time, the beginning value as well as the ending value of an index must be assumed. Although this is really hard, if the right values are assumed and stochastic logistic equation is used, stationary state can be found. The following statement is proved by the theorem about stationary states given in the background chapter. The following strategy can be used as the trading strategy although there is still a lot of space for further research.

\section{REFERENCES}

[1] Hairer, M., Ergodicity of stochastic differential equations driven by fractional Brownian motion. Annals of Probability, 33, pp. 703-758, 2003. http://dx.doi.org/10.1214/009117904000000892

[2] Dung, N.T., On delayed logistic equation driven by fractional Brownian motion, 7(3), pp. 2-7, 2011.

[3] Carmona, P., Coutin, L. \& Montseny, G., Stochastic integration with respect to fractional Brownian motion. Annales de l'Institut Henri Poincare (B) Probability and Statistics, 39(1), pp. 27-68, 2003.

[4] Guillouzic, S., L'Heureux, I. \& Longtin, A., Small delay approximation of stochastic delay differential equations, Physical Review E, 59, pp. 3970-3982, 1999. American Physiological Society, Oxford. http://dx.doi.org/10.1103/physreve.59.3970

[5] Dung, N.T., A class of fractional stochastic differential equations. Vietnam Journal of Mathematics, 36(3), pp. 271-279, 2009.

[6] Ferrante, M. \& Rovira, C. Stochastic delay differential equations driven by fractional Brownian motion with Hurst parameter H > 112. Bernoulli, 12(1), pp. 85-100, 2006.

[7] Ferrante, M. \& Rovira, C., Convergence of delay differential equations driven by fractional Brownian motion. Journal of Evolution Equations, 10(4), pp. 761-783, 2010. http://dx.doi.org/10.1007/s00028-010-0069-8

[8] Dung, N.T., Semimartingale approximation of Fractional Brownian motion and its applications. Computers and Mathematics With Applications, 61, pp. 1844-1854, 2011. http://dx.doi.org/10.1016/j.camwa.2011.02.013

[9] Coutin, L., An introduction to stochastic calculus with respect to fractional Brownian motion, In Seminaire de Probabilities XL, Springer Verlag: Berlin, Heidelberg, pp. 3-65, 2007. 
[10] Dung, N.T. \& Thao, T.H., An approximate approach to fractional stochastic integration and its application. Brazilian Journal of Probability and Statistics, 24(1), pp. 57-67, 2010. http://dx.doi.org/10.1214/08-bjps013

[11] Tiele, T.A., Multiscaling and stock market efficiency in China, Review of Pacific Basin Financial Markets and Policies, 7(4), pp. 1-22, 2014.

[12] Chiang, T.C., Lao, L. \& Xue, Q., Empirical evidence of co-movements between China and global stock markets. 26th Australasian Finance and Banking Conference, http://papers.ssrn. com/sol3/papers.cfm?abstract_id=2315040, 2012.

[13] Cont, R., Long range dependence in financial markets. Centre for financial mathematics, Ecole Polytechnique, Fractals in Engineering 2005, France, pp 159-179, http://www.proba.jussieu.fr/ pageperso/ramacont/papers/FE05.pdf, 2011.

[14] Chen, L., On the chaotic dynamic analysis of Chinese stock market, Conference for Young Computer Scientists, IEEE publisher, Hunan, Young Computer Scientists, 2008. ICYCS 2008. The 9th International Conference for Computer Scientists, pp. 3011-3015, 2012.

[15] Nualart, D., Differential equations driven by fractional Brownian motion. Collectanea Mathematica, 53(1), pp. 55-81, 2002.

[16] Federal Reserve Bank of St. Louis, https://www.stlouisfed.org/. 